\title{
Generalizations of Functionally Generated Portfolios with Applications to Statistical Arbitrage
}

\author{
Winslow Strong* \\ ETH Zürich, Department of Mathematics \\ CH-8092 Zürich, Switzerland \\ winslow.strong@math.ethz.ch
}

\begin{abstract}
The theory of functionally generated portfolios (FGPs) is an aspect of the continuous-time, continuouspath Stochastic Portfolio Theory of Robert Fernholz. FGPs have been formulated to yield a master equation - a description of their return relative to a passive (buy-and-hold) benchmark portfolio serving as the numéraire. This description has proven to be analytically very useful, as it is both pathwise and free of stochastic integrals. Here we generalize the class of FGPs in several ways: (1) the numéraire may be any strictly positive wealth process, not necessarily the market portfolio or even a passive portfolio; (2) generating functions may be stochastically dynamic, adjusting to changing market conditions through an auxiliary continuous-path stochastic argument of finite variation. These generalizations do not forfeit the important tractability properties of the associated master equation. We show how these generalizations can be usefully applied to scenario analysis, statistical arbitrage, portfolio risk immunization, and the theory of mirror portfolios.
\end{abstract}

Keywords: Stochastic Portfolio Theory, functionally generated portfolio, statistical arbitrage, portfolio theory, portfolio immunization, mirror portfolio, master equation

Mathematics Subject Classification: $91 \mathrm{G} 10 \cdot 60 \mathrm{H} 30$

JEL Classification: G11 · C60

\section{Introduction and background}

Functionally generated portfolios (FGPs) were introduced by Robert Fernholz in [5,7], see also 8, 10]. They have historically been constructed by selecting a deterministic generating function that takes the market

${ }^{*}$ The author gratefully acknowledges financial support from the National Centre of Competence in Research "Financial Valuation and Risk Management" (NCCR FINRISK), project D1 (Mathematical Methods in Financial Risk Management), as well as from the ETH Foundation. 
portfolio as its argument. They are notable for admitting a description of their performance, relative to a passive (buy-and-hold) numéraire, that is both pathwise and free of stochastic integrals. This description is known as the master equation, and is a useful tool for portfolio analysis and optimization.

In markets that are uniformly elliptic and diverse [11], and more generally those markets with sufficient intrinsic volatility 9, FGPs yield explicit portfolios that are arbitrages relative to the market portfolio (although see [18] for an alternative diverse market model that is compatible with no-arbitrage). In more general equity market models, FGPs are useful for exploiting certain statistical regularities, such as the stability of the distribution of capital over time 8, 10, and the non-constancy of the rate of variance of logprices as a function of sampling interval [12. These FGP-derived portfolios are best described as statistical arbitrages 12, since they exploit the aforementioned statistical regularities in the data to achieve favorable risk-return profiles. One of the main attractions of the techniques presented in this paper will undoubtedly be towards characterizing and optimizing such statistical arbitrage portfolios.

This paper is organized as follows: Section 2 defines a market model typical of those used in Stochastic Portfolio Theory. Section 3 extends the class of FGPs from its historical definition by allowing an arbitrary wealth process to serve as numéraire, rather than restricting it to be the market portfolio or a more gen-

eral passive portfolio. Generating functions are also extended to accommodate continuous-path auxiliary stochastic arguments of finite variation. Section 3.2 highlights the usefulness of FGPs for scenario analysis. Sections 3.3 and 3.4 provide some characterization of equivalence classes of FGPs in general, and in the case of passive numéraires, respectively. Section 4 explores two approaches of applying FGPs to statistical arbitrage: extending the original idea from $[12$ and using a new construction based on quadratic generating functions. Section 5 presents a method of immunizing a given FGP from certain market risks, while keeping it in the family of FGPs. Section 6 extends the notion of mirror portfolios introduced in 11 and analyzes their asymptotic behavior. Section 7 summarizes the results, and poses some remaining challenges to tackle for the theory of FGPs.

\section{Setting and definitions}

The market consists of $n$ processes with prices $X_{t}=\left(X_{1, t}, \ldots, X_{n, t}\right)^{\prime}$, one of which may be a money market account. $X$ lives on a filtered probability space $\left(\Omega, \mathcal{F}, \mathbb{F}:=\left\{\mathcal{F}_{t}\right\}_{t \geq 0}, \mathbb{P}\right)$, which supports a $d$-dimensional Brownian motion $W_{t}$, where $d \geq n$. All processes introduced are assumed to be progressive with respect to $\mathbb{F}$, which may be strictly bigger than the Brownian filtration.

Most of the analysis herein will take place on $\log$ prices $L_{t}:=\log X_{t}$. The dynamics of $L$ are given by

$$
d L_{t}=\gamma_{t} d t+\sigma_{t} d W_{t}
$$

where $\gamma$ and $\sigma$ are $\mathbb{F}$-progressive and satisfy

$$
\begin{aligned}
\sum_{i=1}^{n} \int_{0}^{t}\left(\left|\gamma_{i, s}\right|+a_{i i, s}\right) d s & <\infty, \quad \forall t \\
\text { where } a_{i j, t}: & =\left[\sigma_{t} \sigma_{t}^{\prime}\right]_{i j}=\frac{d}{d t}\left\langle\log X_{i}, \log X_{j}\right\rangle_{t}, \quad 1 \leq i, j \leq n,
\end{aligned}
$$


and $\sigma_{t}$ takes values in $\mathbb{R}^{n \times d}, d \geq n, \forall t \geq 0$. Throughout, all equalities hold merely almost surely. The notation $\mathbf{1}:=(1, \ldots, 1)$ is used, where the dimensionality should be clear from the context.

Definition 2.1. A portfolio $\pi$ on $X$ is an $\mathbb{F}$-progressively measurable $\mathbb{R}^{n}$-valued process satisfying

$$
\begin{array}{r}
\int_{0}^{t}\left(\left|b_{\pi, s}\right|+\pi_{s}^{\prime} a_{s} \pi_{s}\right) d s<\infty, \quad \forall t \geq 0 \\
\text { and } \sum_{i=1}^{n} \pi_{i, t}=1, \quad \forall t \geq 0 .
\end{array}
$$

The wealth process $V^{v, \pi}$ arising from investment according to $\pi$ is given by

$$
\begin{aligned}
d \log V_{t}^{v, \pi} & =\gamma_{\pi, t} d t+\sigma_{\pi, t} d W_{t}, \\
V_{0}^{v, \pi} & =v \in(0, \infty), \\
\text { where } \sigma_{\pi}: & =\pi^{\prime} \sigma, \\
\gamma_{\pi, t}: & =\pi_{t}^{\prime} \gamma_{t}+\gamma_{\pi, t}^{*}, \\
\text { and } \gamma_{\pi, t}^{*}: & =\frac{1}{2}\left(\sum_{i=1}^{n} \pi_{i, t} a_{i i, t}-\pi_{t}^{\prime} a_{t} \pi_{t}\right) .
\end{aligned}
$$

The process $\gamma_{\pi}^{*}$ is called the excess growth rate, and plays an important role in Stochastic Portfolio Theory 8. 10. To ease notation, we will use $V_{t}^{\pi}:=V_{t}^{1, \pi}$ and often omit the subscript " $t$ " when referring to processes.

Remark 2.2. There is no need for $X$ to be restricted to be the directly tradeable assets of a market. Some components may also be wealth processes of portfolios on the tradeable assets, e.g. $X_{i}=V^{\nu}$, where $\nu$ is a portfolio on the $m<n$ tradeable assets. A weighting of $\pi_{i}$ in $X_{i}$ is equivalent to (additional) weights of $\pi_{i}\left(\nu_{1}, \ldots, \nu_{m}\right)$ in the tradeable assets (beyond what $\pi$ already explicitly specifies for those assets). This flexibility may seem needlessly confusing, but it is useful when portfolios are constructed with consideration to certain market segments - e.g. value, growth, large/small cap, sectors, countries, etc. It's also used in Section 4 below. Distinguishing between the directly tradeable assets and portfolios assembled on them can be important if and when transaction costs and liquidity constraints are taken into consideration, as turnover and leverage may be vastly different. Those issues are beyond the scope of this paper.

The following notation will prove useful:

$$
\begin{aligned}
X^{\rho}: & =\frac{X}{V^{\rho}} \\
L^{\rho}: & =\log X^{\rho}, \\
a_{i j}^{\rho}: & =\left[\sigma \sigma^{\prime}\right]_{i j}=\frac{d}{d t}\left\langle L_{i}^{\rho}, L_{j}^{\rho}\right\rangle, \\
& =a_{i j}-[a \rho]_{j}-[a \rho]_{i}+a_{\rho \rho}, \\
a_{\pi \pi}: & =\sigma_{\pi} \sigma_{\pi}^{\prime}=\pi^{\prime} a \pi=\frac{d}{d t}\left\langle\log V^{\pi}, \log V^{\pi}\right\rangle, \\
a_{\pi \pi}^{\rho}: & =\pi^{\prime} a^{\rho} \pi=\frac{d}{d t}\left\langle\log \left(\frac{V^{\pi}}{V^{\rho}}\right), \log \left(\frac{V^{\pi}}{V^{\rho}}\right)\right\rangle=a_{\rho \rho}^{\pi} .
\end{aligned}
$$


The numéraire invariance property of $\gamma_{\pi}^{*}$ holds for arbitrary portfolios $\pi$ and $\rho$ :

$$
\gamma_{\pi}^{*}=\frac{1}{2}\left(\sum_{i=1}^{n} \pi_{i} a_{i i}^{\rho}-a_{\pi \pi}^{\rho}\right),
$$

$((3.5)$ of 10$)$, and in particular, since $a_{\rho \rho}^{\rho}=0$ by 2.2 ,

$$
\gamma_{\rho}^{*}=\frac{1}{2} \sum_{i=1}^{n} \rho_{i} a_{i i}^{\rho} .
$$

When $\rho$ is exclusively invested in the money market, then discounted quantities will be denoted by $\hat{X}:=X^{\rho}$, $\hat{V}^{\pi}:=V^{\pi} / X^{\rho}$, etc. Note also that in this case $a^{\rho}=a$.

\section{Generalizations of functionally generated portfolios}

Functionally generated portfolios were first introduced in [5,7], see also 8, 10], and the recent extension [15]. There are two generalizations presented here:

(i) The numéraire in the master equation may be arbitrary. Previously, it had been taken to be the market portfolio or some other passive (buy-and-hold) portfolio.

(ii) Generating functions may take stochastic arguments, which here we limit to finite-variation processes.

\subsection{Stochastic generating functions and arbitrary numéraires}

It is natural to adjust a portfolio based on changing market conditions. However, FGPs adjust their weights only as a deterministic function of the underlying discounted price process $X^{\rho}$, which doesn't allow for much flexibility. Ideally, one would like to be able to modify the generating function stochastically while preserving a useful pathwise description of relative return that is free from stochastic integrals. As a step in this direction, time-dependent generating functions have already been introduced in 8 . In this section we extend that idea to allow a dependence on auxiliary stochastic processes of finite variation.

With respect to the historical work on portfolio generating functions, we formulate them here in the $\log$ sense with logarithmic argument. Specifically, our generating function $H$ is related to the previous notion of generating function $G$ by $H(y)=\log G\left(e^{y}\right)$. This makes the analysis cleaner for our purposes.

For $H: \mathbb{R}^{n} \times \mathbb{R}^{k} \rightarrow \mathbb{R}$, let $\nabla_{l}$ be the gradient with respect to the first ( $n$-dim) argument of $H, \nabla_{f}$ be the gradient with respect to the second $(k$ - $\operatorname{dim})$ argument, and $D_{l_{i} l_{j}}^{2}, D_{i, j}^{2}$ be the second-order differential operator with respect to components $i$ and $j$ of the first argument of $H$, and generally, respectively.

Theorem 3.1. Let $H \in C^{2,1}\left(\mathbb{R}^{n} \times \mathbb{R}^{k}, \mathbb{R}\right)$ and let $F$ be an $\mathbb{R}^{k}$-valued, $\mathbb{F}$-progressive, continuous-path process of finite variation. Then the portfolio

$$
\pi=\lambda \rho+\nabla_{l} H\left(L^{\rho}, F\right), \quad \text { where } \lambda=1-\mathbf{1}^{\prime} \nabla_{l} H\left(L^{\rho}, F\right)
$$


satisfies the following master equation:

$$
\begin{aligned}
\log \left(\frac{V_{T}^{\pi}}{V_{T}^{\rho}}\right) & =H\left(L_{T}^{\rho}, F_{T}\right)-H\left(L_{0}^{\rho}, F_{0}\right)-\int_{0}^{T}\left[\nabla_{f} H\left(L_{t}^{\rho}, F_{t}\right)\right]^{\prime} d F_{t}+\int_{0}^{T} h_{t} d t, \\
\text { where } h & =\gamma_{\pi}^{*}-\lambda \gamma_{\rho}^{*}-\frac{1}{2} \sum_{i, j=1}^{n} D_{l_{i} l_{j}}^{2} H\left(L^{\rho}, F\right) a_{i j}^{\rho} .
\end{aligned}
$$

When the argument $F$ is not present (or constant), then it may be suppressed. Hence

$$
\pi=\lambda \rho+\nabla H\left(L^{\rho}\right), \quad \text { where } \lambda=1-\mathbf{1}^{\prime} \nabla H\left(L^{\rho}\right),
$$

satisfies the following master equation:

$$
\begin{aligned}
\log \left(\frac{V_{T}^{\pi}}{V_{T}^{\rho}}\right) & =H\left(L_{T}^{\rho}\right)-H\left(L_{0}^{\rho}\right)+\int_{0}^{T} h_{t} d t \\
\text { where } h & =\gamma_{\pi}^{*}-\lambda \gamma_{\rho}^{*}-\frac{1}{2} \sum_{i, j=1}^{n} D_{i j}^{2} H\left(L^{\rho}\right) a_{i j}^{\rho} .
\end{aligned}
$$

Remark 3.2. Except for the change to the log representation, the derivation proceeds analogously to the original master equation [5, Theorem 3.1], which can also be found in 7, 10. The intermediate equations in the earlier derivations are each generalizable to our setting, shown here as Lemmas 3.3 and 3.4 In the special (original) case where $X$ is the total capitalization (shares $\times$ price per share), then normalizing by the initial values so that the \#shares of each asset is 1 and choosing $\rho$ to be the market portfolio results in

$$
\rho=X / \sum_{i=1}^{n} X_{i}=X^{\rho} .
$$

Inserting $L:=\log X$ and this $\rho$ into (3.4) recovers the original master equation. However, in the general setting of this paper, $\rho$ is arbitrary, making $X^{\rho}$ and $\rho$ distinct.

The following two lemmas will be used in the proof of Theorem 3.1 .

Lemma 3.3. For any two portfolios $\pi$ and $\rho$, the following hold

$$
\begin{aligned}
d \log \left(\frac{V^{\pi}}{V^{\rho}}\right) & =\sum_{i=1}^{n} \pi_{i} d L_{i}^{\rho}+\gamma_{\pi}^{*} d t \\
& =\sum_{i=1}^{n} \pi_{i} \frac{d X_{i}^{\rho}}{X_{i}^{\rho}}-\frac{1}{2} a_{\pi \pi}^{\rho} d t
\end{aligned}
$$

Proof. To prove (3.7), by definition

$$
\begin{aligned}
d L_{i}^{\rho} & =d L_{i}-d \log V^{\rho}, \\
& =\gamma_{i} d t+\sigma_{i} d W_{t}-d \log V^{\rho} .
\end{aligned}
$$


Plugging this into the right-hand side of $(3.7)$, we get

$$
\begin{aligned}
\sum_{i=1}^{n} \pi_{i} d L_{i}^{\rho}+\gamma_{\pi}^{*} d t & =\sum_{i=1}^{n} \pi_{i}\left(\gamma_{i} d t+\sigma_{i} d W_{t}\right)-d \log V^{\rho}+\gamma_{\pi}^{*} d t \\
& =d \log V^{\pi}-d \log V^{\rho}
\end{aligned}
$$

To prove (3.8), use

$$
\begin{aligned}
d L_{i}^{\rho} & =\frac{d X_{i}^{\rho}}{X_{i}^{\rho}}-\frac{1}{2} \frac{d\left\langle X_{i}^{\rho}, X_{i}^{\rho}\right\rangle}{\left(X_{i}^{\rho}\right)^{2}}, \\
& =\frac{d X_{i}^{\rho}}{X_{i}^{\rho}}-\frac{1}{2} a_{i i}^{\rho} .
\end{aligned}
$$

Plugging this into (3.7) and expanding $\gamma_{\pi}^{*}$ with the numéraire invariance property 2.3 yields

$$
\begin{aligned}
d \log \left(\frac{V^{\pi}}{V^{\rho}}\right) & =\sum_{i=1}^{n} \pi_{i}\left(\frac{d X_{i}^{\rho}}{X_{i}^{\rho}}-\frac{1}{2} a_{i i}^{\rho} d t\right)+\frac{1}{2}\left(\sum_{i=1}^{n} \pi_{i} a_{i i}^{\rho}-a_{\pi \pi}^{\rho}\right) d t \\
& =\sum_{i=1}^{n} \pi_{i} \frac{d X_{i}^{\rho}}{X_{i}^{\rho}}-\frac{1}{2} a_{\pi \pi}^{\rho} d t
\end{aligned}
$$

Lemma 3.4. For any portfolio $\rho$ on $X$,

$$
\begin{aligned}
& \sum_{i=1}^{n} \rho_{i} d L_{i}^{\rho}=-\gamma_{\rho}^{*} d t \\
& \sum_{i=1}^{n} \rho_{i} \frac{d X_{i}^{\rho}}{X_{i}^{\rho}}=0 .
\end{aligned}
$$

Proof. For the first, use (3.7) with $\pi=\rho$, and for the second use (3.8) with $\pi=\rho$ and $a_{\rho \rho}^{\rho}=0$ from 2.2.

Now we prove Theorem 3.1 .

Proof of Theorem 3.1. Initially, consider the case where $F \equiv 1$, and hence the second argument to $H$ may be suppressed. First plug in 3.3 for $\pi$ into 3.7 , and then get 3.9 by applying Lemma 3.4

$$
\begin{aligned}
d \log \left(\frac{V^{\pi}}{V^{\rho}}\right) & =\sum_{i=1}^{n}\left(\lambda \rho_{i}+D_{i} H\left(L^{\rho}\right)\right) d L_{i}^{\rho}+\gamma_{\pi}^{*} d t \\
& =\sum_{i=1}^{n} D_{i} H\left(L^{\rho}\right) d L_{i}^{\rho}+\left(\gamma_{\pi}^{*}-\lambda \gamma_{\rho}^{*}\right) d t
\end{aligned}
$$

where $D_{i}$ is the first derivative operator with respect to the $i$ th component. Expanding $d H\left(L^{\rho}\right)$ gives

$$
\begin{aligned}
d H\left(L^{\rho}\right) & =\sum_{i=1}^{n} D_{i} H\left(L^{\rho}\right) d L_{i}^{\rho}+\frac{1}{2} \sum_{i, j=1}^{n} D_{i j}^{2} H\left(L^{\rho}\right) d\left\langle L_{i}^{\rho}, L_{j}^{\rho}\right\rangle, \\
& =\sum_{i=1}^{n} D_{i} H\left(L^{\rho}\right) d L_{i}^{\rho}+\frac{1}{2} \sum_{i, j=1}^{n} a_{i j}^{\rho} D_{i j}^{2} H\left(L^{\rho}\right) d t .
\end{aligned}
$$


Plugging this into $(3.9)$ yields

$$
d \log \left(\frac{V^{\pi}}{V^{\rho}}\right)=d H\left(L^{\rho}\right)+\left(\gamma_{\pi}^{*}-\lambda \gamma_{\rho}^{*}-\frac{1}{2} \sum_{i, j=1}^{n} a_{i j}^{\rho} D_{i j}^{2} H\left(L^{\rho}\right)\right) d t
$$

proving the case when $F \equiv 1$. For a finite variation $F$ with continuous paths, the Itô-Doeblin formula yields

$$
d H\left(L^{\rho}, F\right)=\left(d L^{\rho}\right)^{\prime} \nabla_{l} H\left(L^{\rho}, F\right)+(d F)^{\prime} \nabla_{f} H\left(L^{\rho}, F\right)+\frac{1}{2} \sum_{i, j=1}^{n} d L_{i}^{\rho} d L_{j}^{\rho} D_{l_{i} l_{j}}^{2} H\left(L^{\rho}, F\right),
$$

which when combined with 3.10 proves the theorem.

While adding an auxiliary stochastic process $F$ causes FGPs to lose some elegance and tractability (comparing (3.4) to (3.2)), the extra flexibility gained can be useful in practice. For example, $F$ may be factors that inform portfolio construction, such as those of Fama and French [3, 4], fundamental economic data such as bond yields or stock market diversity $6,8,10,11$, or information extracted from Twitter feeds 2 .

Remark 3.5 (Generalizations). It's possible to remove the restrictions on $F$ - that it's finite variation and has continuous paths - to derive a more general master equation, but this would make the correction term $\int_{0}^{T}\left[\nabla_{f} H\left(L_{t}^{\rho}, F_{t}\right)\right]^{\prime} d F_{t}$ of 3.2 more complex. A continuous $F$ of finite variation is sufficient for the applications that follow, so we do not pursue these extensions here.

If a portfolio $\nu$ satisfies (3.4) in place of $\pi$, then $V^{\nu}$ must be indistinguishable from $V^{\pi}$. Hence, any differences between $\nu$ and $\pi$ are not meaningful in the context of wealth-creation. However, there are portfolios obeying generalizations of the master equation for which $H \notin C^{2}$, such as the class presented in Theorem 4.1 of [16].

The following example looks at the strategy of switching from an initial FGP to a subsequent one at a stopping time. The overall portfolio is an example of a stochastic FGP.

Example 3.6 (Stochastic switching between FGP\&1). Let $H_{1}$ and $H_{2}$ be arbitrary generating functions and let $H$ be

$$
H(y, i):=i H_{1}(y)+(1-i) H_{2}(y), \quad y \in \mathbb{R}^{n}, i \in \mathbb{R} .
$$

For an arbitrary stopping time $\tau, H\left(\cdot, \mathbf{1}_{t \leq \tau}\right)$ is a stochastic generating function (i.e. a function $H$ meeting the requirements of Theorem 3.1 with auxiliary $F$ ) which will generate an FGP that switches at $\tau$ from $\pi^{(1)}$ generated by $H_{1}$ to $\pi^{(2)}$ generated by $H_{2}$. This type of portfolio was used by Banner and D. Fernholz in [1] for constructing arbitrages relative to the market portfolio at arbitrarily short deterministic horizons $T>0$ in a class of market models including volatility-stabilized markets 9 . Those models have also been shown to admit functionally generated relative arbitrage over sufficiently long time horizons 9 . However, there is a horizon before which functionally generated relative arbitrage is not possible, regardless of the choice of generating function 19 . This example shows that relative arbitrages exist on arbitrarily short horizons within the class of FGPs that have stochastic generating functions.

\footnotetext{
${ }^{1}$ The author wishes to thank Radka Pickova for suggesting this idea.
} 


\subsection{Pathwise returns for scenario analysis}

One of the main analytical benefits of the master equation is that it is free of stochastic integrals. When formulas for portfolio returns contain stochastic integrals, then correct analysis may be counterintuitive, and incorrect analysis may be intuitively appealing, as the following example demonstrates.

Example 3.7. Consider two portfolios on horizons $0 \leq t \leq T$ : Let $\pi$ be a long-only constant-weight portfolio so that $\pi_{t}=p, 0 \leq t \leq T$, and let $\tilde{\pi}$ be a passive (buy-and-hold) portfolio starting from the same initial allocation $\tilde{\pi}_{0}=p$. Consider the set $A_{\varepsilon}:=\left\{\omega \in \Omega \mid\left\|\tilde{\pi}_{t}(\omega)-\pi_{t}(\omega)\right\|<\varepsilon, 0 \leq t \leq T\right\}$. In some models for $X$, (e.g. geometric Brownian motion), $P\left(A_{\varepsilon}\right)>0, \forall \varepsilon>0$. Recalling that the usual description of the wealth process of an arbitrary portfolio $\theta$ is

$$
\log V_{T}^{\theta}=\int_{0}^{T} \gamma_{\theta, t} d t+\int_{0}^{T} \theta_{t}^{\prime} \sigma_{t} d W_{t}
$$

then we may write

$$
\left|\log V_{T}^{\pi}-\log V_{T}^{\tilde{\pi}}\right| \leq \int_{0}^{T}\left|\gamma_{\pi, t}-\gamma_{\tilde{\pi}, t}\right| d t+\left|\int_{0}^{T}\left(\pi_{t}-\tilde{\pi}_{t}\right)^{\prime} \sigma_{t} d W_{t}\right| .
$$

If $\gamma$ and $\sigma$ are bounded, then it is tempting to make the erroneous conclusion that

$$
\lim _{\varepsilon \rightarrow 0} \operatorname{esssup}_{\omega \in A_{\varepsilon}}\left|\log V_{T}^{\pi}(\omega)-\log V_{T}^{\tilde{\pi}}(\omega)\right|=0 .
$$

The erroneous conclusion might be stated in words as:

If two portfolios remain sufficiently close to each other, then their returns must be close.

The erroneous conclusion can be avoided by noting that $\pi$ and $\tilde{\pi}$ are functionally generated by generating functions $H(y)=\sum_{i=1}^{n} p_{i} y_{i}$, and $\tilde{H}(y)=\log \left(\sum_{i=1}^{n} p_{i} e^{y_{i}-l_{i}}\right)$, respectively, where $l=L_{0} \in \mathbb{R}^{n}$. Comparing their wealth processes via their master equations gives the pathwise equation

$$
\log V_{T}^{\pi}-\log V_{T}^{\tilde{\pi}}=H\left(L_{T}\right)-H\left(L_{0}\right)-\left(\tilde{H}\left(L_{T}\right)-\tilde{H}\left(L_{0}\right)\right)+\int_{0}^{T} \gamma_{p, s}^{*} d s
$$

If the covariance $a$ is uniformly elliptic (there exists $u>0$ such that $y^{\prime} a_{t} y \geq u\|y\|^{2}$, for all $y \in \mathbb{R}^{n}, t \geq 0$ ), and if $p_{i}>0, \forall i$, then $\gamma_{p, t}^{*} \geq u \in(0, \infty), \forall t \geq 0$ [10. Lemma 3.4]. For all $\xi>0$ there exists $\varepsilon>0$ such that $\left\|L_{T}-L_{0}\right\|<\xi$ on $A_{\varepsilon}$. Hence, 3.13 and the continuity of $H$ and $\tilde{H}$ imply that

$$
\lim _{\varepsilon \rightarrow 0} \underset{A_{\varepsilon}}{\operatorname{essinf}}\left\{\log V_{T}^{\pi}-\log V_{T}^{\tilde{\pi}}\right\}=\lim _{\varepsilon \rightarrow 0} \underset{A_{\varepsilon}}{\operatorname{essinf}}\left\{\int_{0}^{T} \gamma_{p, s}^{*} d s\right\} \geq T u,
$$

contradicting (3.12). This correct conclusion is simply obtainable from the pathwise representation of return given by the master equation, but is difficult to arrive at from the traditional representation of return given in (3.11). The upshot is that

Just because two portfolios remain arbitrarily close does not imply that their returns are close. 
More generally, the master-equation description of relative return (3.4 has advantages over the usual description (3.11) for scenario analysis, a technique currently popular in investment management (e.g. see [14]). In the short term, an FGP's performance (particularly its potential for loss) is largely attributable to the first term of (3.4), which is entirely determined by the terminal values of the underlying assets, which themselves are outputs of scenario analysis. Whereas the last term involves the quadratic variation of the path, and is usually easier to estimate with high precision. In contrast, knowledge of the terminal values of the assets is difficult to use in the Itô-integral formulation (3.11).

\subsection{Translation equivariance and numéraire invariance}

Generating functions are overspecified in the following sense. Given a generating function $H$, each member of the equivalence class of generating functions

$$
[H]:=\{\kappa+H \mid \kappa \in \mathbb{R}\}
$$

yields the same function $\nabla H$. Hence, given an arbitrary market $X$ and numéraire $\rho$, any member of $[H]$ yields the same functionally generated portfolio 3.3 .

Definition 3.8. $H: \mathbb{R}^{n} \rightarrow \mathbb{R}$ is translation equivariant if

$$
H(y+\mathbf{1} \kappa)=\kappa+H(y), \quad \forall y \in \mathbb{R}^{n} .
$$

When $H$ is translation equivariant, then $H$ and hence its corresponding FGP depend only on the relative rather than absolute price level. An example of a class of translation-equivariant generating functions is the diversity- $p$ family (see $[8,10]$ ):

$$
H_{p}(y)=\frac{1}{p} \log \left(\sum_{i=1}^{n} \exp \left\{p y_{i}\right\}\right), \quad y \in \mathbb{R}^{n} .
$$

The following is an invariance property of the master equation when $H$ is translation equivariant.

Proposition 3.9. If $H \in C^{2}\left(\mathbb{R}^{n}, \mathbb{R}\right)$ is translation-equivariant, then $\lambda=0$ in (3.3), and (3.4) exhibits no sensitivity to numéraire choice. Specifically, for arbitrary portfolios $\rho, \phi, \eta$, and $\nu$,

$$
\begin{aligned}
\log \frac{V_{T}^{\pi}}{V_{T}^{\rho}} & =H\left(L_{T}^{\rho}\right)-H\left(L_{0}^{\rho}\right)+\int_{0}^{T} h_{t} d t, \\
\text { where } \pi & =\nabla \log H\left(L^{\phi}\right), \\
\text { and } h & =\gamma_{\pi}-\frac{1}{2} \sum_{i, j=1}^{n} D_{i j}^{2} H\left(L^{\eta}\right) a_{i j}^{\nu} .
\end{aligned}
$$

Proof. Starting with (3.4), we show that when $H$ is translation equivariant, then $\lambda$ of 3.3 is identically 0 :

$$
\begin{aligned}
\frac{\partial}{\partial \kappa} H(y+\mathbf{1} \kappa) & =\frac{\partial}{\partial \kappa}(H(y)+\kappa)=1 \\
\frac{\partial}{\partial \kappa} H(y+\mathbf{1} \kappa) & =\sum_{i=1}^{n} \frac{\partial\left(y_{i}+\kappa\right)}{\partial \kappa} D_{i} H(y+\mathbf{1} \kappa)=\sum_{i=1}^{n} D_{i} H(y+\mathbf{1} \kappa)=1-\lambda .
\end{aligned}
$$


Next, we show that $a^{\rho}$ may be formally replaced with $a^{\nu}$ in 3.5 . We note that

$$
\sum_{i=1}^{n} D_{i j}^{2} H(y)=D_{j} \sum_{i=1}^{n} D_{i} H(y)=D_{j}(1)=0, \quad \forall y \in \mathbb{R}^{n} .
$$

Using this and the form 2.2 for $a^{\rho}$ yields

$$
\begin{aligned}
\sum_{i, j=1}^{n} D_{i j}^{2} H a_{i j}^{\rho} & =\sum_{i, j=1}^{n}\left(a_{i j}-[a \rho]_{j}-[a \rho]_{i}-a_{\rho \rho}\right) D_{i j}^{2} H \\
& =\sum_{i, j=1}^{n} D_{i j}^{2} H a_{i j}-2 \sum_{j}[a \rho]_{j} D_{j} \sum_{i=1}^{n} D_{i} H-a_{\rho \rho} \sum_{i, j=1}^{n} D_{i j}^{2} H \\
& =\sum_{i, j=1}^{n} D_{i j}^{2} H a_{i j} .
\end{aligned}
$$

Reversing the steps shows that $a$ may be replaced with $a^{\nu}$ for arbitrary $\nu$.

It remains to show that $L^{\rho}$ may be replaced with $L^{\eta}$ as the argument to $D^{2} H$ :

$$
D_{i j}^{2} H(y+\mathbf{1} \kappa)=D_{i j}^{2}(H(y)+\kappa)=D_{i j}^{2} H(y), \quad \forall y \in \mathbb{R}^{n}, \forall \kappa \in \mathbb{R}
$$

Since $L^{\rho}=L-\log V^{\rho}$, this implies that

$$
h=\gamma_{\pi}^{*}-\frac{1}{2} \sum_{i, j=1}^{n} D_{i j}^{2} H\left(L^{\rho}\right) a_{i j}^{\nu}=\gamma_{\pi}^{*}-\frac{1}{2} \sum_{i, j=1}^{n} D_{i j}^{2} H\left(L^{\eta}\right) a_{i j}^{\nu} .
$$

Remark 3.10. An FGP can be thought of as a $\Delta$-hedge for its generating function, as it eliminates stochastic integrals from $d H\left(L^{\rho}\right)$. Under this interpretation, $\lambda$ is the position taken in the numéraire with the leftover money in the portfolio. The numéraire $\rho$ sets a stochastic relative price level for $V^{\pi}$ and $L$ in the master equation. When $H$ is translation equivariant, then the corresponding FGP and wealth process have no sensitivity to the price level, as can be seen by Proposition 3.9 and

$$
H\left(L^{\rho}\right)=H\left(L-1 \log V^{\rho}\right)=H(L)-\log V^{\rho},
$$

which simplifies 3.15 to

$$
\log V_{T}^{\pi}=H\left(L_{T}\right)-H\left(L_{0}\right)+\int_{0}^{T} h_{t} d t
$$

Generally, each choice of numéraire results in a unique master equation. But when $H$ is translation equivariant, then there's no excess exposure to the numéraire (beyond what's needed to $\Delta$-hedge $H$ ), making the master equations arising from different numéraire choices trivial translations of the same one equation (3.17).

\subsection{Passive numéraires and gauge freedom}

The historical work on FGPs $[5,7,8,10]$ takes $X$ as the total capitalizations and the numéraire $\rho$ as the market portfolio, leading to $\rho=X^{\rho}$ (see 3.6), as in Remark 3.2. The important property of the market 
portfolio that was exploited in those works was its passivity on $X$ (see Definition 3.11). In the traditional case, the equality of $\rho$ and $X^{\rho}$ means that $\sum_{i=1}^{n} X_{i}^{\rho}=1$, hence the generating function need not be defined on all of $\mathbb{R}^{n}$. In this section we explore more generally to what extent a passive numéraire allows a reduction of the domain of the generating function.

Definition 3.11. A portfolio $\rho$ is passive if there exists a constant $s \in \mathbb{R}^{n}$, called the shares, such that

$$
V^{\rho}=s^{\prime} X \quad \text { and } \quad \rho_{i}=\frac{s_{i} X_{i}}{s^{\prime} X}, \quad 1 \leq i \leq n .
$$

Passive portfolios are untraded after the initial allocation, so are unaffected by transaction costs and other liquidity concerns. Generally, the $X_{i}$ are unbounded from above, so in order that $V^{\rho}>0$ is guaranteed, we assume henceforth that any passive portfolio is long-only. That is, that $s \in[0, \infty)^{n} \backslash\{0\}$.

When the numéraire $\rho$ is passive, then a generating function need not be defined on all of $\mathbb{R}^{n}$, as $X^{\rho}$ will be confined to a hyperplane. Let $s \in[0, \infty)^{n} \backslash\{0\}$ be the constant vector of shares such that $V^{\rho}=s^{\prime} X$. Then,

$$
s^{\prime} X^{\rho}=\frac{s^{\prime} X}{V^{\rho}}=\sum_{i=1}^{n} \rho_{i}=1 .
$$

Thus, $X^{\rho}$ is confined to a hyperplane of codimension 1 , and it should be sufficient to define $H$ on

$$
E_{s}^{n}:=\left\{y \in \mathbb{R}^{n} \mid \sum_{i=1}^{n} s_{i} e^{y_{i}}=1\right\} .
$$

However, Theorem 3.1 uses the Cartesian coordinate system, which is quite convenient, so we sacrifice some generality and require generating functions to be defined on a neighborhood in $\mathbb{R}^{n}$ containing $E_{s}^{n}$.

Definition 3.12. Let $s \in[0, \infty)^{n} \backslash\{0\}$ and let the passive portfolio $\rho$ be given by $\rho_{i}=s_{i} X_{i} / s^{\prime} X, 1 \leq i \leq n$. A $\rho$-generating function is a function $H \in \mathcal{C}^{2}(U, \mathbb{R})$, where $U$ is a neighborhood in $\mathbb{R}^{n}$ containing $E_{s}^{n}$.

When $\rho$ is the market portfolio and $X$ is the total capitalization, then $s \propto 1$. In 8, Proposition 3.1.14], where generating functions are specified as $G(x):=\exp \{H(\log x)\}$, the following equivalence is demonstrated: Generating functions $H_{1}$ and $H_{2}$ generate the same portfolio if and only if $H_{1}-H_{2}$ is constant on $E_{1}^{n}$. This generalizes to general passive portfolios as follows.

Proposition 3.13. Let $\rho$ be passive with corresponding shares $s \in[0, \infty)^{n} \backslash\{0\}$. Let $H_{1}$ and $H_{2}$ be two $\rho$-generating functions defined on a neighborhood $U$ containing $E_{s}^{n}$. Then $H_{1}$ and $H_{2}$ generate the same portfolio for any realization of $X$ if and only if $H_{1}-H_{2}$ is constant on $E_{s}^{n}$.

Proof. Let $\pi^{j}$ be the portfolio generated by $H_{j}, j \in\{1,2\}$. The condition $\pi_{i}^{1}=\pi_{i}^{2}, 1 \leq i \leq n$ for all realizations of $X$ is equivalent by 3.3 to the following holding $\forall y \in E_{s}^{n}$ :

$$
\begin{gathered}
\left(1-\sum_{j=1}^{n} D_{j} H_{1}(y)\right) \rho_{i}+D_{i} H_{1}(y)=\left(1-\sum_{j=1}^{n} D_{j} H_{2}(y)\right) \rho_{i}+D_{i} H_{2}(y), \quad 1 \leq i \leq n, \\
\Longleftrightarrow \frac{s_{i} e^{y_{i}}}{\sum_{k=1}^{n} s_{k} e^{y_{k}}} \sum_{j=1}^{n} D_{j}\left(H_{1}(y)-H_{2}(y)\right)=D_{i}\left(H_{1}(y)-H_{2}(y)\right), \quad 1 \leq i \leq n, \\
\Rightarrow\left(s_{1} e^{y_{1}}, \ldots, s_{n} e^{y_{n}}\right) \propto \nabla\left(H_{1}(y)-H_{2}(y)\right), \quad 1 \leq i \leq n,
\end{gathered}
$$


Differentiating the equation determining the surface $E_{s}^{n}$ shows that 3.18 is equivalent to $\nabla\left(H_{1}-H_{2}\right)$ being orthogonal to $E_{s}^{n}$, hence equivalent to $H_{1}-H_{2}$ being constant on $E_{s}^{n}$. Conversely, if $H_{1}-H_{2}$ is constant on $E_{s}^{n}$, then 3.18 holds. Since by definition $\rho \propto\left(s_{1} e^{y_{1}}, \ldots, s_{n} e^{y_{n}}\right)$, then from $3.3 \pi \pi^{1}-\pi^{2} \propto \rho$. But $\rho$ is long-only, and $\sum_{i=1}^{n}\left(\pi_{i}^{1}-\pi_{i}^{2}\right)=0$. Thus $\pi^{1}=\pi^{2}$.

The gauge freedom implied by Proposition 3.13, specifically by (3.18), is that if $H$ generates $\pi$, then

$$
H_{f}(y):=f\left(\sum_{i=1}^{n} s_{i} e^{y_{i}}\right)+H(y), \quad y \in U \supset E_{s}^{n},
$$

also generates $\pi$, for any $f \in \mathcal{C}^{2}((0, \infty), \mathbb{R})$. This gauge freedom allows one to make any convenient choice for $f$ in order to simplify calculations. The $\rho$-generating functions and general generating functions have their associated respective equivalence classes:

$$
\begin{aligned}
{[H]_{\rho} } & :=\left\{f\left(s^{\prime} e^{\cdot}\right)+H(\cdot) \mid f \in \mathcal{C}^{2}((0, \infty), \mathbb{R})\right\}, \quad \text { where } s_{i}:=\frac{\rho_{i, 0}}{X_{i, 0}}, 1 \leq i \leq n, \\
{[H] } & :=\{H+\kappa \mid \kappa \in \mathbb{R}\} .
\end{aligned}
$$

Each member of a given $[H]_{\rho}$ or $[H]$ is equivalent for the purposes of $\rho$-FGPs, or generally FGPs, respectively.

\section{Statistical arbitrage}

\subsection{Long-short statistical arbitrage with FGPs}

The paper 12 of R. Fernholz and C. Maguire introduces an idea for a statistical arbitrage strategy in markets where the realized rate of variance of $\log$ market prices depends on the sampling interval. The general idea is to take a long position in an FGP that is rebalanced over a time interval corresponding to a high variance rate, hedged with a short position in an FGP generated from the same generating function, but rebalanced over a different time interval corresponding to a low variance rate. Statistical arbitrage profits accrue from the different rates of variance-capture (the $h$ of (3.5) - named so because these terms are directly proportional to the variance rate. Because the long and short FGPs have the same generating function, their corresponding $H$ terms of 3.4 are identical, providing an effective hedge for each other.

The data presented in 12 indicate that for 2005, the variance rate was significantly higher at higher sampling frequencies intradaily for large-cap US equities. The authors looked at rebalancing the long component at 90second intervals and rebalancing the short component once a day. These choices of rebalancing intervals were ad hoc, not the output of an optimization problem. In this section we develop general performance formulas for such long-short statistical arbitrages, creating a framework for optimizing the selection of generating function and rebalancing intervals.

We will show that the growth rate of the statistical arbitrage portfolio always has the quadratic form

$$
\gamma_{\pi}=A \kappa-B \kappa^{2}, \quad A, B>0
$$

where $\kappa$ is the leverage factor, that is, the weight invested in the long portfolio. Hence, there is a level 
of leverage $\bar{\kappa}=A / B$ above which the portfolio tends to shrink in value rather than grow. The leverage $\check{\kappa}=A /(2 B)=\bar{\kappa} / 2$ gives the maximal growth rate of $\check{\gamma}_{\pi}=A^{2} /(4 B)$.

To estimate the performance of the strategy described in [12, we use $X=\left(X_{1}, X_{2}, X_{3}, \ldots, X_{3+m}\right)$, where $X_{3}$ is the money market, and $X_{3+1}, \ldots, X_{3+m}$ are the risky assets that are directly tradeable on the market (e.g. the equities). $X_{1}$ and $X_{2}$ are the values of long-only portfolios on $\left(X_{4}, \ldots, X_{3+m}\right)$. The statistical arbitrage portfolio is an FGP specified on the submarket $\left(X_{1}, X_{2}, X_{3}\right)$ (for more detail, see Remark 2.2). In 12 constant-weight FGPs are considered, where the overall portfolio $\pi$ has $\pi_{1}=-\pi_{2}=\kappa \in(0, \infty)$, and $\pi_{3}=1$. This portfolio is functionally generated by the generating function $H(y)=\kappa\left(y_{1}-y_{2}\right)$, so 3.4 yields

$$
\log \hat{V}_{T}^{\pi}=\kappa\left(\hat{L}_{1, T}-\hat{L}_{1,0}-\left[\hat{L}_{2, T}-\hat{L}_{2,0}\right]\right)+\int_{0}^{T} h_{s} d s
$$

The statistical arbitrage construction uses $X_{1}$ and $X_{2}$ as discretely-traded approximations to the same (continuously traded) FGP, starting from $X_{1,0}=X_{2,0}$. The portfolios differ only in their rebalancing interval. Their values are approximated with the master equation, as if they were continuously-traded. This approximation has been shown empirically to be accurate for diversity- and entropy-weighted FGP approximations that are rebalanced merely once a month [8, Chapter 6]. $X_{1}$ and $X_{2}$ have the same generating functions, $H_{1}=H_{2}$, so under this approximation their values differ only through their variance-capture terms, $h_{1}$ and $h_{2}$, which differ only because rebalancing occurs at different intervals, and hence different effective variance-rates may (and do in practice) apply. The resulting approximation is

$$
\log \hat{V}_{T}^{\pi} \approx \kappa\left(\underline{H_{1}\left(L_{T}\right)-H_{1}\left(L_{0}\right)}+\int_{0}^{T} h_{1, s} d s-\left[\underline{H_{2}\left(L_{T}\right)-H_{2}\left(L_{0}\right)}+\int_{0}^{T} h_{2, s} d s\right]\right)+\int_{0}^{T} h_{s} d s .
$$

The overall portfolio $\pi$ is a constant-weight FGP, hence has $h=\gamma_{\pi}^{*}$ from (3.4). Therefore,

$$
\log \hat{V}_{T}^{\pi} \approx \int_{0}^{T}\left[\kappa\left(h_{1, s}-h_{2, s}+\frac{a_{11}-a_{22}}{2}\right)-\frac{\kappa^{2}}{2} a_{(1,-1)(1,-1)}\right] d s
$$

This has the quadratic form of 4.1. To estimate the parameters, we approximate the stochastic quantities as constants, and plug in the values of their sample estimators. We can identify

$$
\begin{aligned}
& A=h_{1}-h_{2}+\frac{1}{2}\left(a_{11}-a_{22}\right), \\
& B=\frac{1}{2} a_{(1,-1)(1,-1)} .
\end{aligned}
$$

In 12 the FGP chosen to give the value processes $X_{1}$ and $X_{2}$ was the equal-weight portfolio on large cap US equities, specifically those in the S\&P500 and/or Russell 1000 in 2005. The annualized sample averages for that year were $a_{11}=.0683, a_{22}=.0423, a_{(1,-1)(1,-1)}=1.69 \times 10^{-7}, h_{1}=0.0341, h_{2}=0.0211$. $^{2}$ These result in $A=0.0260, B=8.45 \times 10^{-8}, \check{\kappa}=\frac{A}{2 B}=1.54 \times 10^{5}$, and $\check{\gamma}_{\pi}=2.00 \times 10^{3}$ (base $e$ ).

While $\check{\kappa}$ is not of the order of magnitude usually seen in portfolio construction, it must be remembered that it is not a weight for investment into equities, but rather into a long-short combination of two very diverse portfolios that are very similar nearly always. At the beginning of each day, the long and short portfolios

\footnotetext{
${ }^{2}$ These are the numbers from 12 , after transforming standard deviations to variances and annualizing all numbers: $a_{11}=$ $250 * 0.000273, a_{22}=250 * 0.000169, a_{(1,-1)(1,-1)}=250 *\left(\frac{0.0026}{100}\right)^{2}, h_{1}=0.0341, h_{2}=0.0211$.
} 
are equal, so the net position in each equity starts at 0. Each FGP is an equal-weight portfolio in about 1000 equities. If we approximate each initial weight as $10^{-3}$ and use a leverage factor of $\kappa=1.5 \times 10^{5}$, then an isolated $1 \%$ intraday price movement of a particular equity induces a change in net weight of 1.5 in that equity. While this is still an unrealistically leveraged portfolio, it is much closer to a reasonable order of magnitude considering that it would be offset by similarly sized positions of opposite sign.

Despite the above remark, the amount of leverage involved in the $\check{\kappa}$ portfolio is prohibitive due to the realities of equity markets that lie outside of the framework of this paper, such as price jumps, margin requirements, transaction costs, short-selling fees, liquidity constraints, etc. It is these factors then that become the limiting ones for the level of leverage to use in seeking profitability from a statistical arbitrage portfolio of this type. A more plausible level of leverage of $\kappa=1 \times 10^{3}$ results in $\gamma_{\pi}=26$, still orders of magnitude outside the realm of documented performance.

\subsection{Quadratic generating functions}

This section again considers a market whose log asset prices have a variance rate varying with the sampling interval (e.g. Figure 1). Taylor expanding the generating function term in the master equation (3.4) yields

$$
\log \left(\frac{V_{T}^{\pi}}{V_{T}^{\rho}}\right)=\left(\Delta_{T} L^{\rho}\right)^{\prime} \nabla H\left(L_{0}^{\rho}\right)+\frac{1}{2}\left(\Delta_{T} L^{\rho}\right)^{\prime} D^{2} H\left(L_{0}^{\rho}\right) \Delta_{T} L^{\rho}+R_{T}+\int_{0}^{T} h_{t} d t
$$

where $\Delta_{t} L:=L_{t}-L_{0}$,

and $R_{T}$ is the remainder term. For a given path $\omega$, there is a sufficiently short time horizon such that an FGP generated by an analytic $H$ behaves nearly as if it were generated by a quadratic $H$ :

$$
\begin{aligned}
H(y) & =-\frac{1}{2}(y-l)^{\prime} c(y-l)+p^{\prime} y, \quad y, l, p \in \mathbb{R}^{n}, c \in \mathbb{R}^{n \times n}, \\
\Rightarrow \nabla H(y) & =-c(y-l)+p \\
\Rightarrow D^{2} H(y) & =-c .
\end{aligned}
$$

Since statistical arbitrage portfolios are generally rebalanced quite frequently (intradaily), the above motivates consideration of FGPs having quadratic $H$ for application in statistical arbitrage. We assume that the investor has no information, or at least does not wish to speculate, on the drifts of $L^{\rho}$. If this is the case, then it makes no sense for him to take on unnecessary exposure to the $\left(\Delta L_{T}^{\rho}\right)^{\prime} \nabla H\left(L_{0}^{\rho}\right)$ term in 4.3 . This term can be eliminated by selecting an $H$ satisfying $\nabla H\left(L_{0}^{\rho}\right)=0$.

To accomplish this initial hedge, take $p=0$ and $l=L_{0}^{\rho}$ in 4.4 . Then

$$
\begin{aligned}
\log \left(\frac{V_{T}^{\pi}}{V_{T}^{\rho}}\right) & =-\frac{1}{2}\left(\Delta_{T} L_{i}^{\rho}\right)^{\prime} c\left(\Delta_{T} L_{i}^{\rho}\right)+\frac{1}{2} \sum_{i, j} c_{i j} \int_{0}^{T} a_{i j, s}^{\rho} d s+\int_{0}^{T}\left(\gamma_{\pi, s}^{*}-\lambda \gamma_{\rho, s}^{*}\right) d s, \\
\pi & =\lambda \rho-c \Delta_{T} L^{\rho} .
\end{aligned}
$$

For simplicity in illustrating the idea, we restrict the investment to one risky asset (possibly a wealth process of a more general portfolio, as in the previous section) and one locally risk-free asset, i.e. a money market account, which will be the numéraire. The procedure is readily generalizable to a bigger market, with the 
cost being solving and optimizing vector instead of scalar equations. In this setting $\gamma_{\rho}^{*}=0, L^{\rho}=\hat{L}$, and $\pi=\lambda \rho-c \Delta \hat{L}$. Since the money market discounted with itself has value one for all time, then $H$ only need be prescribed on the risky discounted asset's $\log$ price, $\hat{L}$, and hence $c$ is a scalar. The log wealth is

$$
\begin{aligned}
\log \hat{V}_{T}^{\pi} & =\frac{1}{2} c\left(\int_{0}^{T} a_{s} d s-\left(\Delta_{T} \hat{L}\right)^{2}\right)+\int_{0}^{T} \gamma_{\pi, s}^{*} d s \\
& =\frac{1}{2}\left[\int_{0}^{T}\left(c a_{s}+a_{s}\left[c \Delta_{s} \hat{L}\right]_{i}-c^{2}\left[\Delta_{s} \hat{L}\right]^{2} a_{s}\right) d s-c\left(\Delta_{T} \hat{L}\right)^{2}\right] .
\end{aligned}
$$

To proceed, we take an expectation, assume Brownian integrals are martingales, and approximate some time-dependent parameters as constants. This simplifies the model, allowing for easy fitting to data:

$$
\begin{aligned}
E\left[a_{t}\right] & \approx: a \\
E \Delta_{s} \hat{L} & =E\left[\int_{0}^{s} \hat{\gamma}_{u} d u\right] \approx: s \hat{\gamma}, \\
E\left[\left(\Delta_{s} \hat{L}\right)^{2}\right] & =: A_{s} .
\end{aligned}
$$

Note: $a$ is best interpreted as $A_{t} / t$ at the $t$ at which trading actually occurs. Using the above in (4.5) yields

$$
\begin{aligned}
E \log \left(\hat{V}_{T}^{\pi}\right) & \approx \frac{1}{2}\left[a c T+a c \hat{\gamma} \int_{0}^{T} s d s-a c^{2} \int_{0}^{T} A_{s} d s-c A_{T}\right], \\
& =\frac{T a}{2}\left(c\left[1-\frac{A_{T}}{T a}+\frac{\hat{\gamma} T}{2}\right]-c^{2} v(T)\right), \\
\text { where } v(T): & =\frac{1}{T} \int_{0}^{T} A_{s} d s .
\end{aligned}
$$

By assumption, $A_{t} / a t$ is not identically 1 . If its deviation from 1 is not substantially greater than $|\hat{\gamma} t / 2|$ for some $t>0$, then our exposure to $\hat{\gamma}$ results in large risk for little gain. In any case, we are ignorant of the drift, so we drop it and are left with

$$
\frac{1}{T} E \log \left(\hat{V}_{T}^{\pi}\right) \approx \frac{a}{2}\left(c\left[1-\frac{A_{T}}{T a}\right]-c^{2} v(T)\right) .
$$

All that is needed is the variogram for $\hat{L}$, from which the other quantities are easily derived. The $c$ that maximizes the expected $\log$ growth by horizon $T$ is

$$
\check{c}_{T}=\frac{1}{2 v(T)}\left(1-\frac{A_{T}}{T a}\right) .
$$

This yields a maximal expected log-growth rate of

$$
\frac{1}{T} E \log \left(\hat{V}_{T}^{\pi}\right)=\frac{a}{8 v(T)}\left(1-\frac{A_{T}}{T a}\right)^{2} .
$$

Empirically, the quantity $A_{T} / T$ tends to a constant for large $T$, thus $v(T)=\mathcal{O}(T)$ for $T \rightarrow \infty$. This means that the growth rate tends to 0 as $T \rightarrow \infty$. The question then arises of what the optimal period $\check{T}$ is for restarting this strategy. This can be obtained by maximizing (4.7) as a function of $T$. 


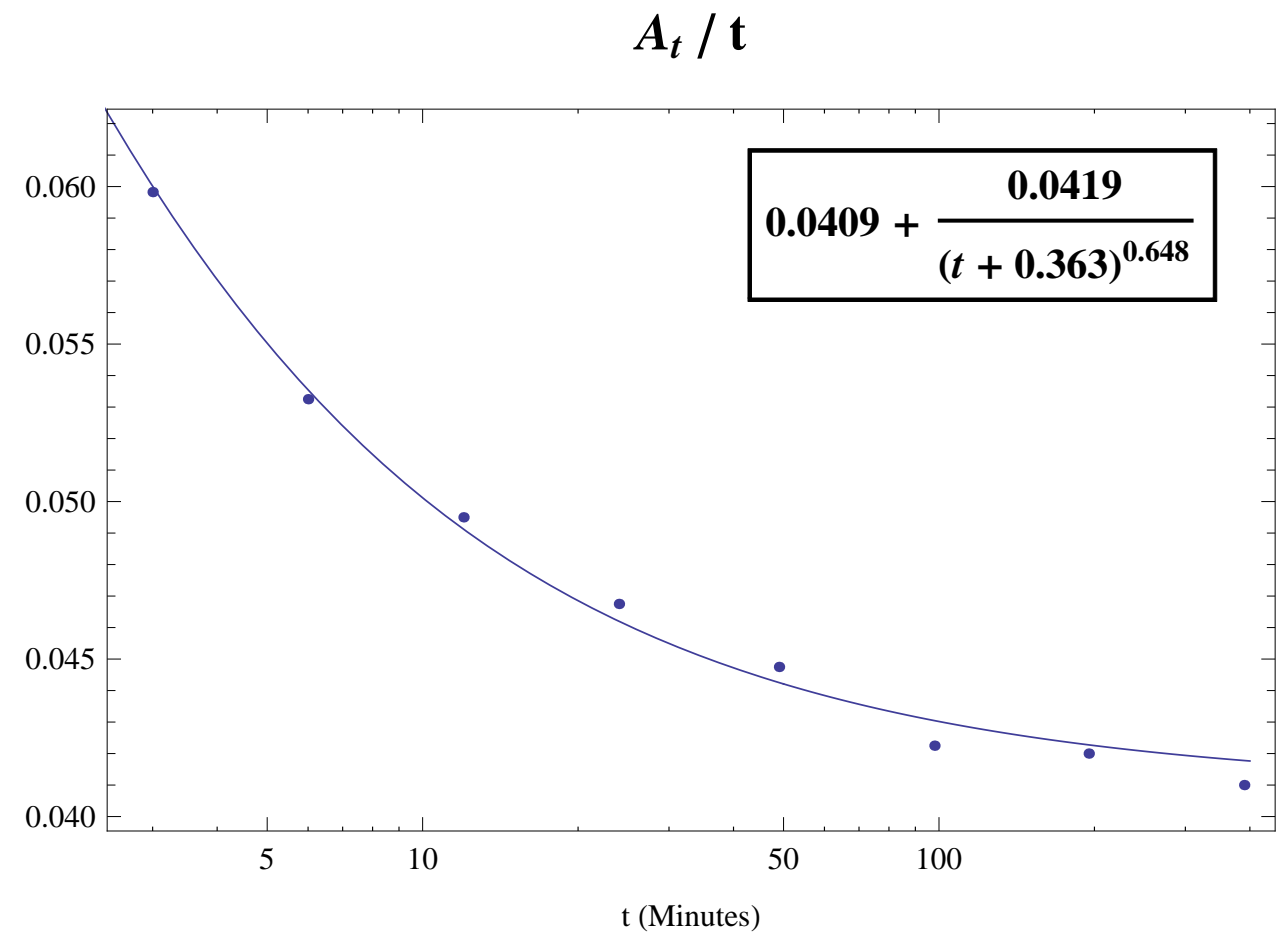

Figure 1: Our fit to the annualized variogram of US-large cap stocks from [12, Figure 1].

Conjecture 4.1. Rather than restarting the portfolio after a given time period, it may be better to solve an optimal control problem, restarting when the price of the risky asset wanders sufficiently far from its origin.

A positive feature of both the methodologies of Section 4 is that they are entirely data-driven, and depend only on variance measurements, which can be estimated in practice with high precision. Of course if the data suggests a parametric model, then the added structure could be additionally exploited.

Example 4.2. We apply a quadratic generating function to the data from [12, i.e. of an equal-weight portfolio on large cap US equities in 2005. The variogram (Figure 1) is fitted to

$$
\frac{A_{t}}{t}=C+\frac{U}{(t+B)^{k}}
$$

The form was chosen for fitting fairly well and also because $A$ has a closed-form antiderivative, yielding

$$
v(T) \equiv \frac{1}{T} \int_{0}^{T} A_{t} d t=\frac{C T}{2}+U k \frac{(B+T)^{1-k}([1-k] T-B)+B^{2-k}}{T(2-k)(1-k)} .
$$

Assuming, as in Section 4.1 that our (fastest) rebalancing frequency is 1.5 minutes, then effectively $a=$ $A_{1.5} / 1.5=.0683$ (annualized). From 4.7 we numerically obtain $\check{T}=7.13$ minutes, leading to $\check{c}(\check{T})=$ $5.8 \times 10^{4}$, and a maximal rate of log return of 244 (base $e$, per year). This is an order of magnitude lower than $\hat{\gamma}_{\pi}$ obtained from the same data via the methodology in Section 4.1. This may be explained by the approximation made in 4.2 breaking down under substantial leverage. Another factor may be that our 


\section{Rate of Log Return}

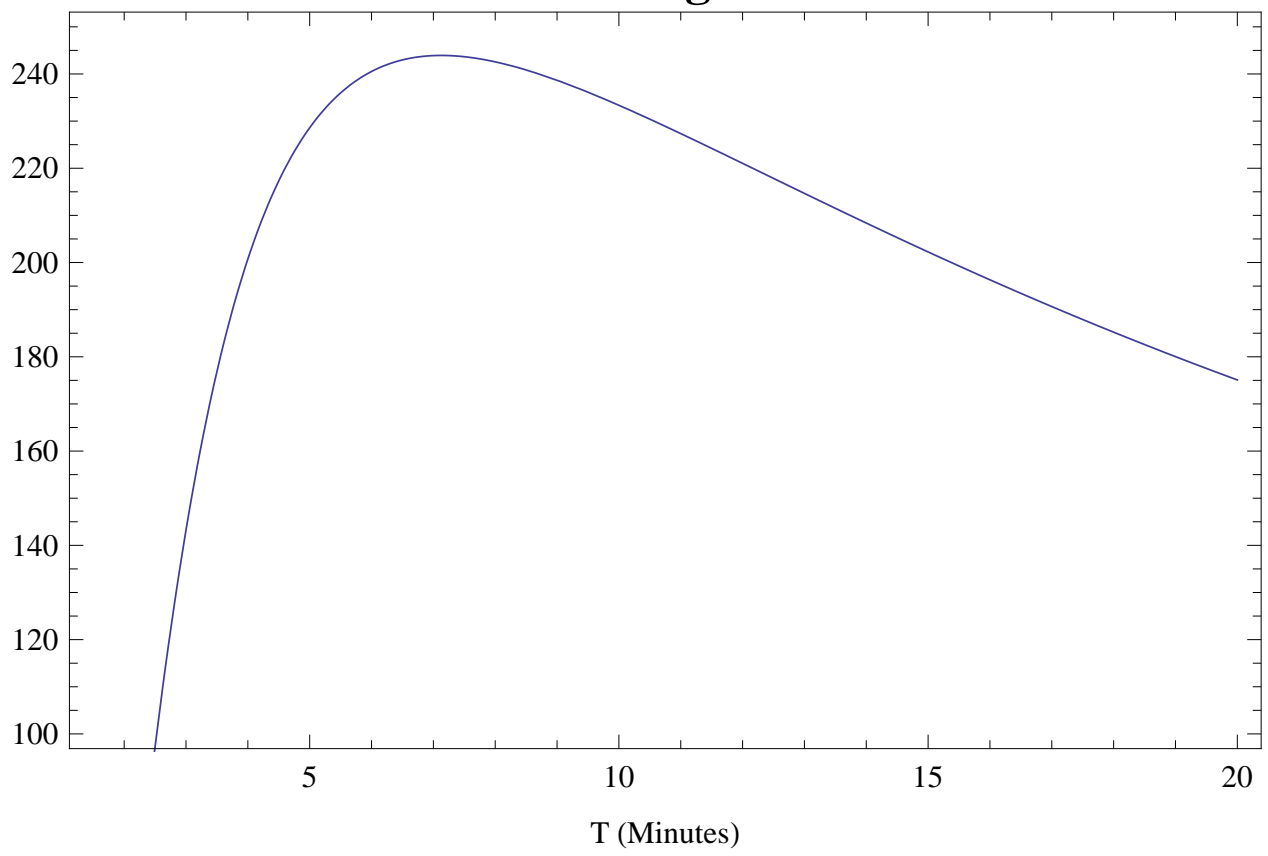

Figure 2: The optimal annualized rate of log-return as a function of horizon in Example 4.2

dropping the drift term $\hat{\gamma} T / 2$ of 4.6 underestimates the return of the quadratic FGP: Since $A_{T} / T<a$, then conditioned on a price movement, a large drift in the opposite direction is typical, which the portfolio implicitly bets on.

\section{Portfolio immunization}

Suppose that we have selected a generating function that is appealing, except that it produces a generated portfolio that is exposed, relative to the numéraire, to risk factors that we would rather remain unexposed to. For example, we may wish to avoid taking on numéraire risk by maintaining zero excess exposure to it. One way to remove unwanted risk exposure is to modify the initial generating function, only in so far as to make it invariant to changes in the argument along the direction of given risk factors. To be more concrete, suppose that $H$ is the initial generating function, and that $\beta^{1}, \ldots, \beta^{K}$ are each continuous-path finite variation processes in $\mathbb{R}^{n}$ satisfying

$$
\left(\beta_{t}^{k}\right)^{\prime} \beta_{t}^{j}=\delta_{k j}, \quad 1 \leq k, j \leq K, \quad \forall t \geq 0 .
$$

The orthonormal set of random vectors $\left\{\beta_{t}^{1}, \ldots, \beta_{t}^{K}\right\}$ spans the subspace in $\mathbb{R}^{n}$ that we would like to immunize the generated portfolio's performance to at time $t$. That is, we would like to find a generating function $\tilde{H}$, similar to $H$, except also obeying

$$
\left(\beta_{t}^{k}\right)^{\prime} \nabla \tilde{H}_{t}=0, \quad \forall t \geq 0
$$


For this to hold, $\tilde{H}$ will need to be stochastic, via taking $\beta:=\left\{\beta_{i}^{k}\right\}_{1 \leq k \leq n}^{1 \leq k}$ as a second argument. Perhaps the most natural way to modify $H$ in order to achieve this is to project $\nabla H$ onto the complement of the span of $\left\{\beta^{1}, \ldots, \beta^{K}\right\}$ and allow that to determine the new function $\tilde{H}$. To that end, let $P^{\perp}(y, b)$ be the projection operator that projects $y$ onto the orthogonal complement of the span of vectors $\left\{b^{k}\right\}^{1 \leq k \leq K}$. It is simplest to specify $P^{\perp}$ in the case where $\left\{b^{k}\right\}$ are orthonormal:

$$
P^{\perp}(y, b)=y-\sum_{k=1}^{K}\left(y^{\prime} b^{k}\right) b^{k}, \quad \text { for }\left(b^{k}\right)^{\prime} b^{j}=\delta_{k j}, 1 \leq k, j \leq K .
$$

Proposition 5.1. Let $\left\{\beta^{1}, \ldots, \beta^{K}\right\}$ be $K \leq n$ finite variation processes in $\mathbb{R}^{n}$ that are mutually orthonormal at all times. The generating function $H$ and generated portfolio

$$
\pi=\lambda \rho+P^{\perp}\left(\nabla H\left(P^{\perp}\left(L^{\rho}, \beta\right)\right), \beta\right), \quad \text { where } \lambda=1-\mathbf{1}^{\prime} P^{\perp}\left(\nabla H\left(P^{\perp}\left(L^{\rho}, \beta\right)\right), \beta\right),
$$

satisfy the following master equation:

$$
\log \left(\frac{V_{T}^{\pi}}{V_{T}^{\rho}}\right)=H\left(P^{\perp}\left(L_{T}^{\rho}, \beta_{T}\right)\right)-H\left(P^{\perp}\left(L_{0}^{\rho}, \beta_{0}\right)\right)+\int_{0}^{T} h_{t} d t-\sum_{k=1}^{K} \int_{0}^{T}\left[\nabla_{b^{k}} H\left(P^{\perp}\left(L_{t}^{\rho}, \beta_{t}\right)\right)\right]^{\prime} d \beta_{t}^{k},
$$

where

$$
\begin{aligned}
h= & \gamma_{\pi}^{*}-\lambda \gamma_{\rho}^{*}-\frac{1}{2}\left(\sum_{i, j=1}^{n} a_{i j}^{\rho} D_{i j}^{2} H\left(P^{\perp}\left(L^{\rho}, \beta\right)\right), \beta\right)-2 \sum_{k=1}^{K}\left(b^{k}\right)^{\prime} a^{\rho} D^{2} H\left(P^{\perp}\left(L^{\rho}, \beta\right)\right) b^{k} \\
& \left.+\sum_{k=1}^{K} \sum_{k^{\prime}=1}^{K}\left(b^{k^{\prime}}\right)^{\prime} a^{\rho} b^{k}\left(b^{k^{\prime}}\right)^{\prime} D^{2} H\left(P^{\perp}\left(L^{\rho}, \beta\right)\right) b^{k}\right), \\
\nabla_{b^{k}} H\left(P^{\perp}(y, b)\right)= & -\left[\left(b^{k}\right)^{\prime} \nabla H\left(P^{\perp}(y, b)\right)\right] y_{i}-\left[\left(b^{k}\right)^{\prime} y\right] D_{i} H\left(P^{\perp}(y, b)\right) .
\end{aligned}
$$

Proof. Define

$$
\begin{aligned}
\tilde{H}: \mathbb{R}^{n} \times \mathbb{R}^{K \times n} & \rightarrow \mathbb{R} \\
\tilde{H}(y, b) & =H\left(P^{\perp}(y, b)\right), \\
\text { where } P^{\perp}(y, b) & =y-\sum_{k=1}^{K}\left(y^{\prime} b^{k}\right) b^{k} .
\end{aligned}
$$

The result is then obtained from a direct application of Theorem 3.1 to $\tilde{H}$. The relevant derivatives are

$$
\begin{aligned}
\nabla_{y} \tilde{H}(y, b)= & \nabla H\left(P^{\perp}(y, b)\right)-\sum_{k=1}^{K}\left[\left(b^{k}\right)^{\prime} \nabla H\left(P^{\perp}(y, b)\right)\right] b^{k} \\
= & P^{\perp}\left(\nabla H\left(P^{\perp}(y, b)\right), b\right) \\
\frac{\partial^{2}}{\partial y_{i} \partial y_{j}} \tilde{H}(y, b)= & D_{i j}^{2} H\left(P^{\perp}(y, b)\right)-\sum_{k=1}^{K} b_{j}^{k}\left[\left(b^{k}\right)^{\prime} D^{2} H\left(P^{\perp}(y, b)\right)\right]_{i}-\sum_{k=1}^{K} b_{i}^{k}\left[\left(b^{k}\right)^{\prime} D^{2} H\left(P^{\perp}(y, b)\right)\right]_{j} \\
& +\sum_{k=1}^{K} \sum_{k^{\prime}=1}^{K} b_{i}^{k} b_{j}^{k^{\prime}}\left(b^{k^{\prime}}\right)^{\prime} D^{2} H\left(P^{\perp}(y, b)\right) b^{k}
\end{aligned}
$$




$$
\frac{\partial}{\partial b_{i}^{k}} \tilde{H}(y, b)=-\left(\left(b^{k}\right)^{\prime} \nabla H\left(P^{\perp}(y, b)\right)\right) y_{i}-\left(\left(b^{k}\right)^{\prime} y\right) D_{i} H\left(P^{\perp}(y, b)\right) .
$$

The characterization of the performance of the immunized FGP given by Proposition 5.1 is not so pretty, but the idea of what has changed from the non-immunized FGP is straightforward. The relative wealth process $\log \left(V^{\pi} / V^{\rho}\right)$ of the generated portfolio of Proposition 5.1 is locally not exposed to changes in $L^{\rho}$ along the linear span of $\left\{\beta^{1}, \ldots, \beta^{K}\right\}$. This can be seen from

$$
\begin{aligned}
\left.\left(\beta^{k}\right)^{\prime} \nabla_{y} H\left(P^{\perp}(y, b)\right)\right|_{\left(L^{\rho}, \beta\right)} & =\left(\beta^{k}\right)^{\prime}(\pi-\lambda \rho), \\
& =\left(\beta^{k}\right)^{\prime} P^{\perp}\left(\nabla H\left(P^{\perp}\left(L^{\rho}, \beta\right)\right), \beta\right), \\
& =0, \quad 1 \leq k \leq K .
\end{aligned}
$$

Example 5.2 (Numéraire exposure). Consider the case where immunization is desired with respect to relative exposure to the numéraire. The appropriate $\beta$ to use to hedge against excess numéraire exposure is 1 less than the "CAPM $\beta$ " (see e.g. 17]). The instantaneous version of this parameter is

$$
\begin{aligned}
\tilde{\beta}_{i, t} & =\frac{\frac{d}{d t}\left\langle\log V^{\rho}, L_{i}^{\rho}\right\rangle_{t}}{\frac{d}{d t}\left\langle\log V^{\rho}\right\rangle_{t}}=\frac{\left[a_{t} r_{t}\right]_{i}-a_{\rho \rho, t}}{a_{\rho \rho, t}}=\frac{\left[a_{t} \rho_{t}\right]_{i}}{a_{\rho \rho, t}}-1, \\
& =\beta_{i, t}^{(\mathrm{CAPM})}-1
\end{aligned}
$$

Although theoretically this instantaneous $\beta$ may not be a continuous-path finite variation process, in practice the instantaneous $\beta$ is not observable, and $\beta$ is typically estimated by time-averaging over some historical time window. The practical and theoretical result of such a time-averaging procedure is a continuous-path finite variation process. For example, the estimator might have the theoretical form

$$
\tilde{\beta}_{i, t}=\frac{\frac{1}{\Delta t} \int_{t-\Delta t}^{t}\left[a_{s} \rho_{s}\right]_{i} d s}{\frac{1}{\Delta t} \int_{t-\Delta t}^{t} a_{\rho \rho, s} d s}-1,
$$

for some $\Delta t>0$. In practice the integrals are approximated by sums of discretely sampled values.

Example 5.3 (Price level). Another possibly desirable immunization is to hedge out any exposure to a rise or fall in the overall price level. This can be done by choosing the constant vector $\beta=n^{-1 / 2} \mathbf{1}$.

\section{Mirror portfolios}

In this section we use generating functions to elaborate some of the properties of mirror portfolios, introduced in [11. In that paper, mirror portfolios were used to construct arbitrages over arbitrarily short time horizons in markets that are both diverse and uniformly elliptic. A passive portfolio that is short any asset is typically inadmissible, due to each asset's price usually being unbounded from above. Hence, flipping the sign of the shares invested in assets (while adjusting the weight in the numéraire so that weights sum to one) does not in general produce a suitable notion of a reflected portfolio. Mirror portfolios accomplish that task.

Definition 6.1. If $\pi$ and $\rho$ are portfolios, then the portfolio

$$
\tilde{\pi}^{[q], \rho}:=q \pi+(1-q) \rho, \quad q \in \mathbb{R},
$$


is called the $q$-mirror of $\pi$ with respect to $\rho$. When $\rho$ is fully invested in the money market, then $\tilde{\pi}^{[q], \rho}=: \tilde{\pi}^{[q]}$, abbreviated the $q$-mirror of $\pi$. For $q=-1, \tilde{\pi}^{[-1], \rho}=: \tilde{\pi}^{\rho}$, called simply the mirror of $\pi$ with respect to $\rho$.

For portfolios $\pi$ and $\rho$, the $q$-mirror of $\pi$ with respect to $\rho$ satisfies 2.1], so is also a portfolio. As an example, if $X_{1}$ is the money market, then the portfolio $e_{i}:=(0, \ldots, 0,1,0, \ldots)$ has the mirror $\tilde{e}_{i}=(2,0, \ldots, 0,-1,0, \ldots)$.

Proposition 6.2. The q-mirror of $\pi$ with respect to $\rho$ is functionally generated from the market $\left(X_{1}, X_{2}\right):=$ $\left(V^{\rho}, V^{\pi}\right)$ by the generating function $H\left(y_{1}, y_{2}\right):=(1-q) y_{1}+q y_{2}$, and thus satisfies

$$
\begin{aligned}
\log V^{\tilde{\pi}^{[q], \rho}} & =(1-q) \log V^{\rho}+q \log V^{\pi}+\int h_{s} d s, \\
\log \left(\frac{V^{\tilde{\pi}^{[q], \rho}}}{V^{\rho}}\right) & =q \log \left(\frac{V^{\pi}}{V^{\rho}}\right)+\int h_{s} d s, \\
\text { where } h=\gamma_{\tilde{\pi}[q], \rho}^{*} & =\frac{1}{2}\left((1-q) a_{11}+q a_{22}-\left[(1-q)^{2} a_{11}+2 q(1-q) a_{12}+q^{2} a_{22}\right]\right) .
\end{aligned}
$$

If, additionally, $\rho$ is the money market, then

$$
\log \hat{V}^{\tilde{\pi}^{[q]}}=q \log \hat{V}^{\pi}+\frac{q(1-q)}{2}\left\langle\log V^{\pi}\right\rangle .
$$

If, additionally, $q=-1$, then

$$
\log \hat{V}^{\tilde{\pi}}=-\log \hat{V}^{\pi}-\left\langle\log V^{\pi}\right\rangle
$$

Proof. $H$ is translation equivariant and $D^{2} H=0$, so we may apply Proposition 3.9 to obtain the first result. The others are easy consequences of plugging in $a_{11}=a_{12}=0$ when $\rho$ is the money market.

The following corollary shows that under typical market conditions a given portfolio $\pi$ or its mirror $\tilde{\pi}$ or both will lose all wealth, asymptotically.

Corollary 6.3. Suppose that both of the following hold:

$$
\liminf _{t \rightarrow \infty} \frac{1}{t}\left\langle\log V^{\pi}\right\rangle_{t}>0, \quad \text { a.s. }
$$

$$
\lim _{t \rightarrow \infty} \frac{\log \log t}{t^{2}}\left\langle\log V^{\pi}\right\rangle_{t}=0, \quad \text { a.s. }
$$

Then

$$
P\left(\left\{\lim _{t \rightarrow \infty} \hat{V}_{t}^{\pi}=0\right\} \bigcup\left\{\lim _{t \rightarrow \infty} \hat{V}_{t}^{\tilde{\pi}}=0\right\}\right)=1 .
$$

Proof. Under 6.3 the law of the iterated logarithm [13, p. 112] implies that

$$
\lim _{t \rightarrow \infty} \frac{1}{t}\left(\log \hat{V}_{t}^{\pi}-\int_{0}^{t} \gamma_{\pi, s} d s\right)=0, \quad \text { a.s. }
$$


since the process inside the parentheses is a continuous local martingale. From this (6.1) yields

$$
\begin{aligned}
& \lim _{t \rightarrow \infty} \frac{1}{t}\left(\log \hat{V}_{t}^{\tilde{\pi}}+\left\langle\log V^{\pi}\right\rangle_{t}+\int_{0}^{t} \gamma_{\pi, s} d s\right)=0, \quad \text { a.s., } \\
& \Rightarrow \lim _{t \rightarrow \infty} \frac{1}{t}\left(\log \hat{V}_{t}^{\pi}+\log \hat{V}_{t}^{\tilde{\pi}}+\left\langle\log V^{\pi}\right\rangle_{t}\right)=0, \quad \text { a.s., }
\end{aligned}
$$

where the second line follows from adding 6.4 to the first. Then by 6.2

$$
\begin{array}{r}
\limsup _{t \rightarrow \infty} \frac{1}{t}\left(\log \hat{V}_{t}^{\pi}+\log \hat{V}_{t}^{\tilde{\pi}}\right)<0, \quad \text { a.s. } \\
\Rightarrow P\left(\left\{\limsup _{t \rightarrow \infty} \frac{1}{t} \log \hat{V}_{t}^{\pi}<0\right\} \bigcup\left\{\limsup _{t \rightarrow \infty} \frac{1}{t} \log \hat{V}_{t}^{\tilde{\pi}}<0\right\}\right)=1 .
\end{array}
$$

Equation 6.1 shows that at least one and possibly both of $\log \hat{V}^{\pi}$ and $\log \hat{V}^{\tilde{\pi}}$ have negative drift at any time when $\left\langle\log V^{\pi}\right\rangle$ is increasing. The preceding corollary shows that a portfolio, its mirror, or possibly both, lose all wealth relative to the money market asymptotically, assuming that the asymptotic local variance rate does not approach 0 . A portfolio whose wealth tends to 0 asymptotically would typically be considered a poor long-term investment. In this sense, "mirroring" a poor investment may still be a poor investment. A concrete example is a market with a risk-free rate of 0 , and one risky asset whose price is a geometric Brownian motion with $\gamma=-\frac{1}{2} \sigma^{2}$. Then full investment in the risky asset loses all wealth asymptotically, as does its mirror, which also has $\operatorname{drift} \tilde{\gamma}=-\frac{1}{2} \sigma^{2}$ by 6.1.

\section{Concluding remarks}

The key analytical benefit of portfolios that are functionally generated is the representation of their return relative to a numéraire via a pathwise master equation free of stochastic integrals. The generalizations of FGPs presented here expand the class of portfolio-numéraire pairs that may be analyzed in this way. The dynamism of FGPs is enhanced by the freedom to incorporate processes having continuous, finite-variation paths as auxiliary arguments to generating functions. This allows FGPs to be sensitive to changing market conditions beyond the price changes of the assets. The main applications that we have shown are (1) direct, intuitive comparison of the performance of FGPs, useful for scenario analysis (Section 3.2), (2) statistical arbitrage based purely on variance data, (3) portfolio immunization, and (4) mirror portfolios analysis.

It is a shortcoming of this work that transaction costs are ignored throughout. They are especially important to the performance of the statistical arbitrage portfolios examined in Section 4 . The inclusion of transaction costs in a tractable way for FGPs in $n$-asset markets is a topic of ongoing research. Due to its complexity, it warrants a separate paper that the author hopes will be forthcoming in the future.

\section{References}

[1] Adrian Banner and D. Fernholz. Short-term relative arbitrage in volatility-stabilized markets. Annals of Finance, 4:445-454, 2008. 
[2] J. Bollen, H. Mao, and X. Zeng. Twitter mood predicts the stock market. Journal of Computational Science, 2(1):1-8, 2011.

[3] F. Eugene and K. French. The cross-section of expected stock returns. Journal of Finance, 47(2):427465, 1992.

[4] E.F. Fama and K.R. French. Common risk factors in the returns on stocks and bonds. Journal of financial economics, 33(1):3-56, 1993.

[5] E. Robert Fernholz. Portfolio generating functions. Technical report, INTECH, 1995. https://ww3. intechjanus. com/Janus/Intech/intech? command=researchListing.

[6] E. Robert Fernholz. On the diversity of equity markets. Journal of Mathematical Economics, 31:393-417, 1999.

[7] E. Robert Fernholz. Portfolio generating functions. In Marco Avellaneda, editor, Quantitative Analysis in Financial Markets, pages 344-364. World Scientific, Singapore, 1999.

[8] E. Robert Fernholz. Stochastic Portfolio Theory. Springer, Berlin, first edition, 2002.

[9] E. Robert Fernholz and Ioannis Karatzas. Relative arbitrage in volatility-stabilized markets. Annals of Finance, 1:149-177, 2005.

[10] E. Robert Fernholz and Ioannis Karatzas. Stochastic Portfolio Theory: an overview. In A. Bensoussan and Q. Zhang, editors, Handbook of Numerical Analysis: Volume XV: Mathematical Modeling and Numerical Methods in Finance, pages 89-167. North Holland, Oxford, 2009.

[11] E. Robert Fernholz, Ioannis Karatzas, and Constantinos Kardaras. Diversity and relative arbitrage in equity markets. Finance and Stochastics, 9:1-27, 2005.

[12] E.R. Fernholz and C Maguire. The statistics of statistical arbitrage. Financial Analysts Journal, 63:46$52,2007$.

[13] Ioannis Karatzas and Steven E. Shreve. Brownian Motion and Stochastic Calculus. Springer, Berlin, second edition, 1991.

[14] J. Mina, J.Y. Xiao, et al. Return to riskmetrics: the evolution of a standard, 2001. New York: RiskMetrics Group.

[15] Soumik Pal and Ting-Kam Leonard Wong. Energy, entropy, and arbitrage. arXiv preprint arXiv:1308.5376, 2013.

[16] Olivier Menoukeu Pamen. A general theorem for portfolio generating functions. Communication on Stochastic Analysis, 5(2):271-283, 2011.

[17] William Sharpe. Portfolio Theory and Capital Markets. McGraw-Hill, 1970.

[18] Winslow Strong and Jean-Pierre Fouque. Diversity and arbitrage in a regulatory breakup model. Annals of Finance, 7:349-374, 2011.

[19] Phillip Whitman. Personal Communication. 